\title{
Clinical Study \\ Effluent Tenascin-C Levels Reflect Peritoneal Deterioration in Peritoneal Dialysis: MAJOR IN PD Study
}

\author{
Ichiro Hirahara, Eiji Kusano, Toshimi Imai, Yoshiyuki Morishita, Makoto Inoue, \\ Tetsu Akimoto, Osamu Saito, Shigeaki Muto, and Daisuke Nagata
}

Division of Nephrology, Department of Internal Medicine, Jichi Medical University, 3311-1 Yakushiji, Shimotsuke, Tochigi 329-0498, Japan

Correspondence should be addressed to Ichiro Hirahara; hirahara@rpf.jp

Received 22 May 2015; Revised 4 September 2015; Accepted 29 October 2015

Academic Editor: Robert Beelen

Copyright (C) 2015 Ichiro Hirahara et al. This is an open access article distributed under the Creative Commons Attribution License, which permits unrestricted use, distribution, and reproduction in any medium, provided the original work is properly cited.

Peritoneal deterioration causing structural changes and functional decline is a major complication of peritoneal dialysis (PD). The aim of this study was to explore effluent biomarkers reflecting peritoneal deterioration. In an animal study, rats were intraperitoneally administered with PD fluids adding $20 \mathrm{mM}$ methylglyoxal (MGO) or $20 \mathrm{mM}$ formaldehyde (FA) every day for 21 days. In the MGO-treated rats, tenascin-C (TN-C) levels in the peritoneal effluents were remarkably high and a cluster of TN-Cpositive mesothelial cells with epithelial-to-mesenchymal transition- (EMT-) like change excessively proliferated at the peritoneal surface, but not in the FA-treated rats. Effluent matrix metalloproteinase-2 (MMP-2) levels increased in both the MGO- and FAtreated rats. In a clinical study at 18 centers between 2006 and 2013, effluent TN-C and MMP-2 levels were quantified in 182 PD patients with end-stage renal disease. Peritoneal function was estimated using the peritoneal equilibration test (PET). From the PET results, the D/P Cr ratio was correlated with effluent levels of TN-C $(\rho=0.57, p<0.001)$ and MMP-2 $(\rho=0.73, p<0.001)$. We suggest that TN-C in the effluents may be a diagnostic marker for peritoneal deterioration with EMT-like change in mesothelial cells in PD.

\section{Introduction}

Peritoneal dialysis (PD) is a treatment for patients with severely reduced or absent renal function. Long-term PD results in peritoneal deterioration, causing structural changes and functional decline such as increased peritoneal solute transport; this leads to ultrafiltration failure of the peritoneal membrane. Peritoneal deterioration may result in the cessation of PD treatment; this complication leads to encapsulating peritoneal sclerosis (EPS) in patients with long-term history of PD for renal failure. EPS is associated with an extremely high mortality rate [1-4]. Safe and effective PD requires monitoring of peritoneal deterioration developing to EPS.

Functional decline of the peritoneum, such as increasing peritoneal transport rate, can be assessed with the peritoneal equilibration test (PET) [4-6]. The transport rate increases with peritoneal deterioration and a higher transporter membrane state is a factor contributing to the occurrence of EPS in patients who have experienced PD treatment [5].
Structural changes of the peritoneum can be examined by biopsy of peritoneal tissue; however, because it is invasive, this method is inappropriate for continuous testing. Only exceptionally skilled clinical pathologists perform cytodiagnosis of mesothelial cells in peritoneal effluents [4]. Easy and noninvasive methods are required to evaluate peritoneal structural changes for diagnosis of peritoneal deterioration.

Some effluent biomarkers, such as matrix metalloproteinase-2 (MMP-2), interleukin-6 (IL-6), hyaluronan, and cancer antigen-125 (CA125), are measured to estimate peritoneal deterioration or progression to EPS during PD [2, 4, 711]. Kaku et al. demonstrated that the correlation coefficient between the peritoneal solute transport rate estimated by the PET and the effluent levels of MMP-2 was higher than those of IL-6, hyaluronate, and CA125 [11]. We also reported that effluent MMP-2 levels were high in the patients with peritoneal deterioration and strongly correlated with the results of the PET [8]. MMP-2 degrades components of the extracellular matrix, such as fibronectin and type IV collagen, 
which comprise the basement membrane. MMP-2 is produced by mesenchymal cells, macrophages, and endothelial cells in the peritoneum; it plays important roles in angiogenesis, epithelial-to-mesenchymal transition (EMT) of mesothelial cells, and migration of cells that promote inflammation or fibroplasia $[2,7,9-15]$. Because they reflect peritoneal deterioration with structural changes and functional decline, effluent MMP-2 levels may be valuable in predicting the occurrence of EPS. Thus, MMP-2 may be the best available indicator of peritoneal deterioration.

Morphologically, mesothelial cells change from polygonal cobblestone-like appearance to a spindle-shaped form in PD [16]. Aguilera et al. reported that the EMT of mesothelial cells may be involved in triggering peritoneal injury with fibrosis in PD patients [17]. In addition, high solute transport of peritoneal membrane is associated with EMT of mesothelial cells [18]. These reports suggest that monitoring EMT of mesothelial cells may enable early diagnosis of peritoneal deterioration.

Tenascin-C (TN-C) is an extracellular matrix glycoprotein formed by hexamers of approximately $300 \mathrm{kDa}$ subunits. Although expression of TN-C is rare in normal adult tissues, high levels are observed in pathological states featuring tissue remodeling, such as inflammation, wound healing, and cancer progression. In particular, $\mathrm{TN}-\mathrm{C}$ is expected as a biomarker for myocarditis or aortic aneurism [19]. In addition, TN-C induces EMT-like change in cancer cells $[20,21]$. As mentioned above, EMT-like change in mesothelial cells is induced at early stage of peritoneal deterioration. TN$\mathrm{C}$ may have potential as an indicator of tissue injury; however, to the best of our knowledge, there are no reports concerning TN-C in PD.

This study aimed to investigate simple and noninvasive methods, to evaluate indicators of peritoneal deterioration used to diagnose early peritoneal deterioration with structural changes and functional decline, such as high solute transport of the peritoneal membrane. Therefore, we examined whether TN-C and MMP-2 in the peritoneal effluents have potential as indicators of peritoneal deterioration.

\section{Methods and Patients}

2.1. Preparation of Animal Models of Peritoneal Injury. Animals used in this study were 5-6-week-old male SpragueDawley (SD) rats weighing approximately 200-250 g (Charles River Japan, Kanagawa, Japan). They were housed in cages in an air-conditioned room, which was maintained at a constant temperature of $23 \pm 2^{\circ} \mathrm{C}$ and relative humidity of $50 \pm 10 \%$. The animals were kept under a 12-hour light/dark cycle and had free access to sufficient pellet food and water.

The PD fluids were prepared by adding $20 \mathrm{mM}$ methylglyoxal (MGO) or $20 \mathrm{mM}$ formaldehyde (FA) to a solution (2.5\% glucose, $100 \mathrm{mM} \mathrm{NaCl}, 35 \mathrm{mM}$ sodium lactate, $2 \mathrm{mM}$ $\mathrm{CaCl}_{2}$, and $0.7 \mathrm{mM} \mathrm{MgCl}_{2}, \mathrm{pH} 5.0$ ), which were then daily sterilized by filtration just before injection. Rats were divided into three groups ( $n=6$ /group) and intraperitoneally administered with the following solutions for 21 days: group $1,100 \mathrm{~mL} / \mathrm{kg} \mathrm{PD}$ fluid without adding MGO or FA; group 2, $100 \mathrm{~mL} / \mathrm{kg}$ PD fluid containing $20 \mathrm{mM} \mathrm{MGO;} \mathrm{group} \mathrm{3,}$
$100 \mathrm{~mL} / \mathrm{kg}$ PD fluid containing $20 \mathrm{mM} \mathrm{FA.} \mathrm{MGO} \mathrm{and} \mathrm{FA}$ concentrations were determined based on previous reports [12-14]. If any solution remained in the peritoneal cavity, it was drained prior to injection. On day 22 after the start of the experiment, $50 \mathrm{~mL} / \mathrm{kg}$ of $\mathrm{PD}$ fluid containing 2.5\% glucose (Midperiq L250, Terumo Co., Tokyo, Japan) was intraperitoneally injected and the drained dialysate was collected 90 minutes later to analyze TN-C and MMP-2 in the effluents. The parietal peritoneum was also sampled for histological analysis.

Adequate attention was paid to maintaining a hygienic environment and to preventing infectious peritonitis in the animals. Furthermore, a sterility test was performed using the dialysate drained on day 22 to check for the presence of aerobic bacteria, anaerobic bacteria, and fungi. All rats were confirmed to be uninfected.

The Institutional Animal Experiment Committee of Jichi Medical University approved the protocol of this animal study. The animal experiments were conducted in accordance with the Institutional Regulations for Animal Experiments and Fundamental Guidelines for the Proper Conduct of Animal Experiments and Related Activities in Academic Research Institutions under the jurisdiction of Japan's Ministry of Education, Culture, Sports, Science and Technology.

2.2. Immunohistological Analysis of Peritoneum in Animal Study. The parietal peritoneum was sampled from the corresponding sites of each rat and fixed with $10 \% \mathrm{FA} / 0.1 \mathrm{M}$ phosphate buffer ( $\mathrm{pH}$ 7.2). The peritoneal specimens were embedded in paraffin to prepare tissue sections with a thickness of $2-3 \mu \mathrm{m}$. To analyze the thickness of the peritoneum, the sections were sliced perpendicular to the peritoneal surface. The peritoneal tissue sections prepared from a paraffin block were dewaxed with xylene. The sections were treated with $0.4 \%$ pepsin $/ 0.01 \mathrm{~N} \mathrm{HCl}$ for 10 minutes at $37^{\circ} \mathrm{C}$, followed by treatment with $0.3 \% \mathrm{H}_{2} \mathrm{O}_{2} /$ methanol for 30 minutes at room temperature. After blocking with $1 \%$ bovine serum albumin for 20 minutes at room temperature, these sections were treated overnight at $7^{\circ} \mathrm{C}$ with a monoclonal antibody against TN-C (Immuno-Biological Laboratories Co., Ltd., Gunma, Japan) at a dilution of 1:500, followed by staining with biotinylated anti-mouse IgG (Dako Cytomation Denmark A/S, Glostrup, Denmark) for 30 minutes at room temperature. The sections were treated with peroxidase-labeled streptavidin (Dako) at a dilution of 1:700 for 30 minutes at room temperature and then peroxidase activity was detected with 3,3'-diaminobenzidine tetrahydrochloride (Sigma, St. Louis, MO, USA). Sections were also counterstained with Meyer's hematoxylin. Negative staining was confirmed by incubation without primary antibody for immunohistochemical staining.

2.3. Analysis of the Drained Dialysate in Animal Study. To explore markers for peritoneal deterioration, TN-C levels in the drained dialysate were quantitatively determined by enzyme-linked immunosorbent assay (ELISA) (ImmunoBiological Laboratories Co., Ltd.). MMP-2 in the dialysate was analyzed by gelatin zymography [7, 12, 13]. In brief, after electrophoresis under nonreducing conditions on $8 \%$ 
polyacrylamide gels containing $1 \mathrm{mg} / \mathrm{mL}$ gelatin, the gels were treated with $2.5 \%$ Triton-100/0.1 M NaCl/50 mM Tris$\mathrm{HCl}(\mathrm{pH} 7.5)$ for 2 hours and incubated for 18 hours at $37^{\circ} \mathrm{C}$ in $50 \mathrm{mM}$ Tris- $\mathrm{HCl}(\mathrm{pH} 7.5) / 10 \mathrm{mM} \mathrm{CaCl}_{2}$. The gels were then stained with 0.1\% Coomassie Brilliant Blue. MMP2 was detected as unstained $64 \mathrm{kDa}$ proteolytic bands in the stained gels. The relative concentrations of MMP- 2 in the dialysate were quantified by scanning proteolytic bands on the zymograms using the ImageJ quantitation software program (National Institutes of Health, Bethesda, MD, USA).

2.4. Patients in Multicenter Clinical Study. PD patients with end-stage renal disease at 18 centers in Japan were analyzed during the period from January 2007 to March 2013. Patients treated with PD for less than 3 months were excluded from the present study, as were those with bacterial peritonitis at the time of the analysis or in the 4 preceding weeks.

Clinical analysis was conducted after receiving approval from the Ethics Committee of Jichi Medical University, and informed consent was obtained from each patient. TN-C in the serum was analyzed in 20 patients from whom informed consent for analysis of the serum had been obtained.

This study was registered as the MAJOR IN PD study (Multicenter Analysis in Japan, ORiginal INdicator of Peritoneal Deterioration) in the University Hospital Medical Information Network-Clinical Trials Registry (UMIN-CTR), which was approved by the International Committee of Medical Journal Editors (number UMIN000010572).

2.5. Peritoneal Equilibration Test (PET) for Human. The peritoneal solute transport rate was assessed by the PET $[4,6]$. Intra-abdominal fluid was drained and PD fluid containing $2.27-2.5 \%$ glucose was intraperitoneally injected. Creatinine (Cr) level of the drained dialysate obtained 4 hours after injection (D) was divided by that of the patient's blood (P) to calculate the $\mathrm{D} / \mathrm{P} \mathrm{Cr}$ ratio. The glucose level of the dialysate obtained 4 hours after injection (D) was divided by that obtained immediately after injection (D0) to calculate the $\mathrm{D} / \mathrm{D} 0$ glucose ratio. The drained dialysate was aliquoted and stored at $-80^{\circ} \mathrm{C}$. It was confirmed that TN-C and MMP-2 were stable at $-80^{\circ} \mathrm{C}$ and were not decomposed by several repeated freeze-thaw cycles.

2.6. Analysis of Biomarker Levels in the Drained Dialysate in Multicenter Clinical Study. The concentrations of TN-C and MMP-2 in the drained dialysate obtained at the PET were measured by EIA (TN-C: Immuno-Biological Laboratories Co., Ltd.; MMP-2: GE Healthcare, Piscataway, NJ, USA).

Serum $\mathrm{TN}-\mathrm{C}$ levels were analyzed and regression lines were calculated based on the power relationship between the molecular weights of $\beta 2$-microglobulin (MW: $11,800 \mathrm{Da}$ ), MMP-3 (MW: 59,000 Da), albumin (MW: 69,000 Da), transferrin (MW: 85,000 Da), and IgG (MW: 150,000 Da) and their measured dialysate/serum (D/S) ratios when plotted on a double-logarithmic scale [8-10]. These proteins were transported from the circulation to the peritoneal cavity by the osmotic pressure of the PD fluid. By interpolation of the molecular weights of TN-C (MW: $1,800,000 \mathrm{Da})$ in the regression equation, the expected $\mathrm{D} / \mathrm{S}$ ratios were calculated, assuming that their concentration in the drained dialysate would be determined by transport only from the circulation.

2.7. Statistical Analysis. Statistical analyses were performed using $\mathrm{R}$ statistical software version 2.15.1 (R Foundation for Statistical Computing). Receiver operating characteristic (ROC) curve analyses were performed to evaluate the diagnostic accuracy of MMP-2 and TN-C. Comparisons between groups were performed by Wilcoxon's test. Relationships between clinical variables and effluent biomarker levels were analyzed by Spearman's correlation coefficient test. Clinical data are expressed as medians with the spread from the 25 th to 75 th percentiles. In the animal experiment, statistical comparisons were conducted by analysis of variance (ANOVA). Animal experimental data are presented as the mean \pm SD. A $p$ value of less than 0.05 was considered to be statistically significant.

\section{Results}

3.1. Animal Experiments. In rats receiving PD fluids containing $20 \mathrm{mM}$ MGO or $20 \mathrm{mM}$ FA, peritoneal thickening significantly increased (Figure 1). These results confirmed that both the MGO- and FA-treated rats had induced peritoneal deterioration with structural changes.

In control rats, a monolayer of mesothelial cells was observed on the surface of the thin peritoneum (Figure 1(a)). In the MGO-treated rats, TN-C was abundant at the surface of the peritoneum where cells excessively proliferated (Figure 1(b)). In the FA-treated rats, there was slight deposition of TN-C at the surface of the peritoneum where cells were lost (Figure 1(c)).

The effluent TN-C levels of the MGO- or FA-treated rats increased approximately 3000 times and 300 times, respectively (Figure 2(a)). The effluent MMP-2 levels of the MGOand FA-treated rats increased 5 and 3.7 times, respectively (Figure 2(b)).

3.2. Relationships between the Peritoneal Solute Transport Rate and TN-C or MMP-2 Levels in the Drained Dialysate Prepared from Patients. A total of 182 PD patients at 18 centers in Japan were analyzed. Patient characteristics are summarized in Table 1. TN-C and MMP-2 levels in the drained dialysate are shown as median (interquartile range) of $7.1 \mathrm{ng} / \mathrm{mL}$ (4.0$12.4 \mathrm{ng} / \mathrm{mL}$ ) and $150 \mathrm{ng} / \mathrm{mL}(103-221 \mathrm{ng} / \mathrm{mL})$, respectively. The levels of TN-C and MMP-2 in the drained dialysate highly correlated with the peritoneal solute transport rate determined by the PET (Table 2). The correlation coefficient between effluent TN-C and MMP-2 levels $(\rho=0.75, p<$ 0.001) was higher than that between these biomarker levels and the results of the PET (Figure 3 and Table 2). These biomarker levels significantly correlated with the numbers of peritonitis episodes, but not PD duration (Figure 4 and Table 3). The relationships between the effluent biomarker levels and the characteristics of the patients are shown in Table 3.

The proportion of high-PET-category patients in the present study was $8 \%$ of PD patients. TN-C and MMP2 levels in the drained dialysate from high-PET-category 


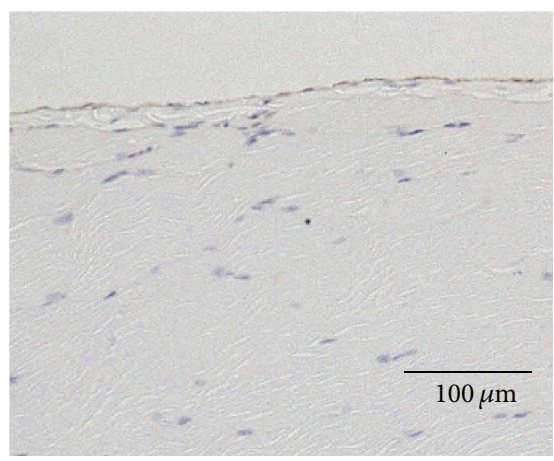

(a)

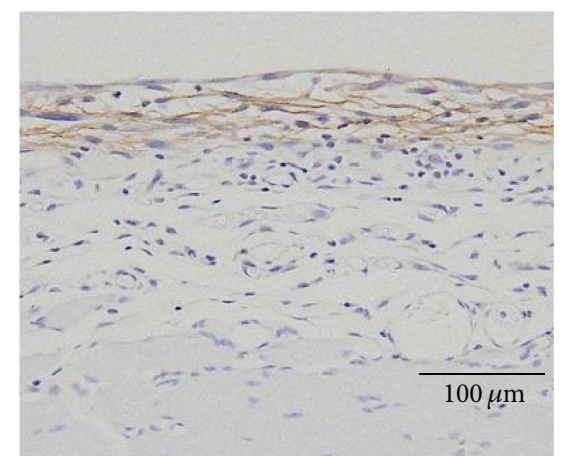

(b)

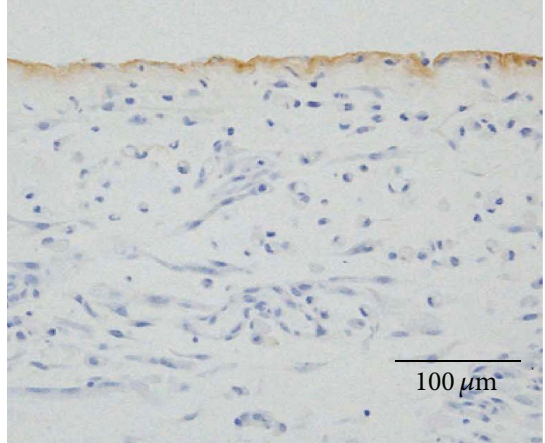

(c)

FIGURE 1: Immunohistopathological findings of peritoneum of the MGO- or FA-treated rats. The parietal peritoneum was analyzed histologically by immune staining with anti-TN-C antibody. (a) Control rat. (b) MGO-treated rat. (c) FA-treated rat. $\times 200$.

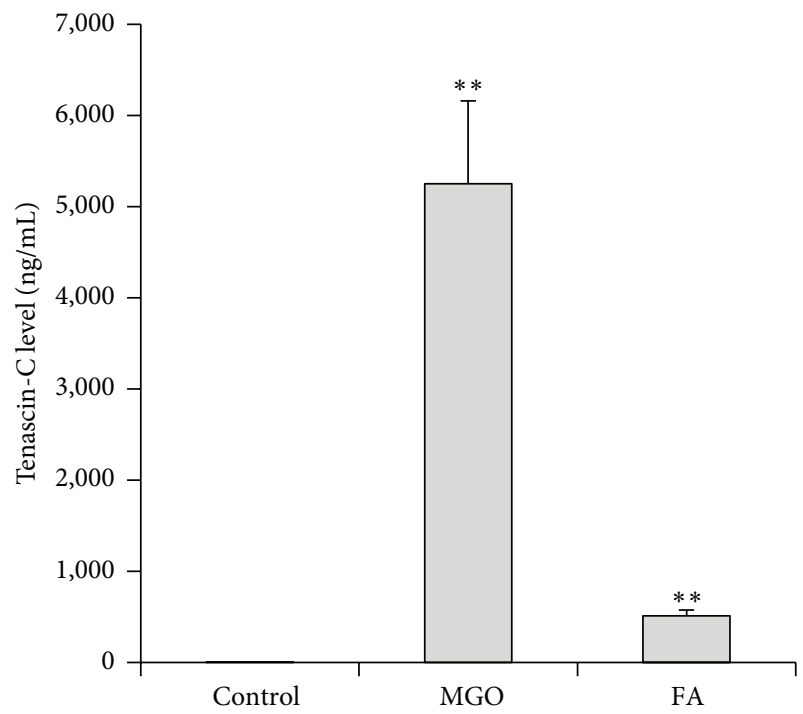

(a)

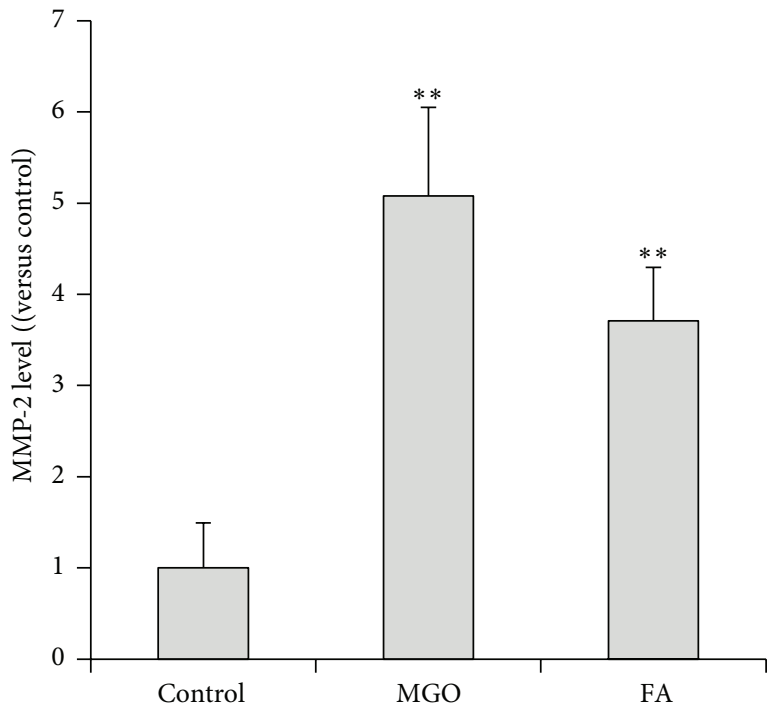

(b)

Figure 2: Effluent levels of TN-C and MMP-2 in the MGO- or FA-treated rats. (a) TN-C levels in the drained dialysate. (b) MMP-2 levels in the drained dialysate. Each column represents the mean \pm SD of 6 rats. ${ }^{* *} p<0.01$ compared with control.

patients were significantly higher than those from non-highPET-category patients (Figure 5). To assess the ability of the biomarkers to diagnose the high category of the PET, ROC curves were constructed. On the ROC curves, the cut-off points of TN-C and MMP-2 for the high category of the PET were $5.7 \mathrm{ng} / \mathrm{mL}$ and $213 \mathrm{ng} / \mathrm{mL}$, respectively (Table 4). The areas under the ROC curve (AUC) for effluent TN-C and MMP-2 levels against the high category of the PET were 0.71 and 0.91 , respectively (Table 4 ).

Serum TN-C levels were analyzed in 20 patients (median age: 58 years, median PD duration: 15 months, 75\% males, $20 \%$ with diabetes). The levels of TN-C in the serum were higher than those in the drained dialysate. The regression line was calculated based on least squares regression analysis between the measured D/S ratios of the serum proteins, such as beta 2-microglobulin, MMP-3, albumin, transferrin, and $\mathrm{IgG}$, and their molecular weights. The measured $\mathrm{D} / \mathrm{S}$ ratio of $\mathrm{TN}-\mathrm{C}$ was also plotted in relation to the molecular weight.
The slope of the regression line represented the size selectivity of the peritoneal membrane; however, the measured $\mathrm{D} / \mathrm{S}$ ratio of TN-C considerably exceeded the regression line $(p<$ $0.01)$. Regression lines for each individual patient were also calculated. Based on each regression line, the expected D/S ratios of TN-C were predicted assuming that their levels in the dialysate would be determined by transport only from the circulation. In all patients, the measured D/S ratios of TN$\mathrm{C}$ considerably exceeded the expected $\mathrm{D} / \mathrm{S}$ ratios calculated based on the regression line of each individual patient (data not shown).

\section{Discussion}

It is important to monitor peritoneal deterioration with structure changes and functional decline, such as an increase of the peritoneal solute transport rate. Functional decline of the peritoneum can be assessed with the PET, but there 
TABLE 1: Characteristics of patients.

\begin{tabular}{lc}
\hline Sex (male/female) & $104 / 78(57 \%$ male $)$ \\
Etiology (nondiabetes/diabetes) & $147 / 35(19 \%$ diabetes $)$ \\
Age (years) & 58 years $(47-66$ years $)$ \\
PD duration (months) & 33 months $(13-59$ months $)$ \\
Peritonitis episode (times) & 0 times $(0-1$ time $)$ \\
D/P Cr & $0.65(0.59-0.71)$ \\
D/D0 glucose & $0.40(0.34-0.44)$ \\
Effluent tenascin-C level & $7.1 \mathrm{ng} / \mathrm{mL}(4.0-12.4 \mathrm{ng} / \mathrm{mL})$ \\
Effluent matrix metalloproteinase-2 level & $150 \mathrm{ng} / \mathrm{mL}(103-221 \mathrm{ng} / \mathrm{mL})$ \\
\hline
\end{tabular}

Data except sex and etiology of renal failure are expressed as medians with interquartile ranges.

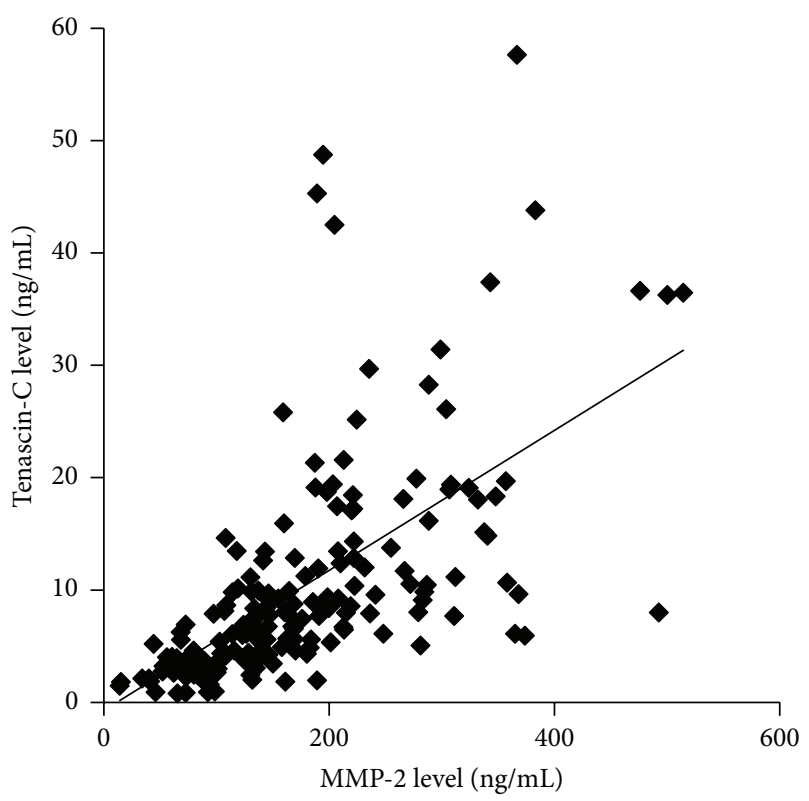

Figure 3: The relationship between TN-C and MMP-2 levels in the drained dialysate.

TABLE 2: Spearman's correlation coefficients between effluent biomarker levels and results of the PET.

\begin{tabular}{lcc}
\hline & Tenascin-C & Matrix metalloproteinase-2 \\
\hline \multirow{2}{*}{ D/P Cr } & $\rho=0.57$ & $\rho=0.73$ \\
& $p<0.001$ & $p<0.001$ \\
\hline \multirow{2}{*}{ D/D0 glucose } & $\rho=-0.43$ & $\rho=-0.63$ \\
& $p<0.001$ & $p<0.001$ \\
\hline
\end{tabular}

$\rho$ values indicate relationships between effluent biomarker levels and results of the PET by Spearman's correlation coefficient.

is no current method to evaluate noninvasively structure changes of the peritoneum. Peritoneal deterioration, whose mechanism is not well known, may develop through multiple factors, such as infectious peritonitis and continuous exposure to unphysiologic $\mathrm{PD}$ fluid with low $\mathrm{pH}$, high osmolarity, high concentration of glucose, and glucose degradation products (GDPs), such as MGO, glyoxal, FA, 3-deoxyglucosone (3-DG), and 3,4-dideoxyglucosone-3-ene (3,4-DGE) [2, 3, 12]. Therefore, we explored the biomarkers for peritoneal

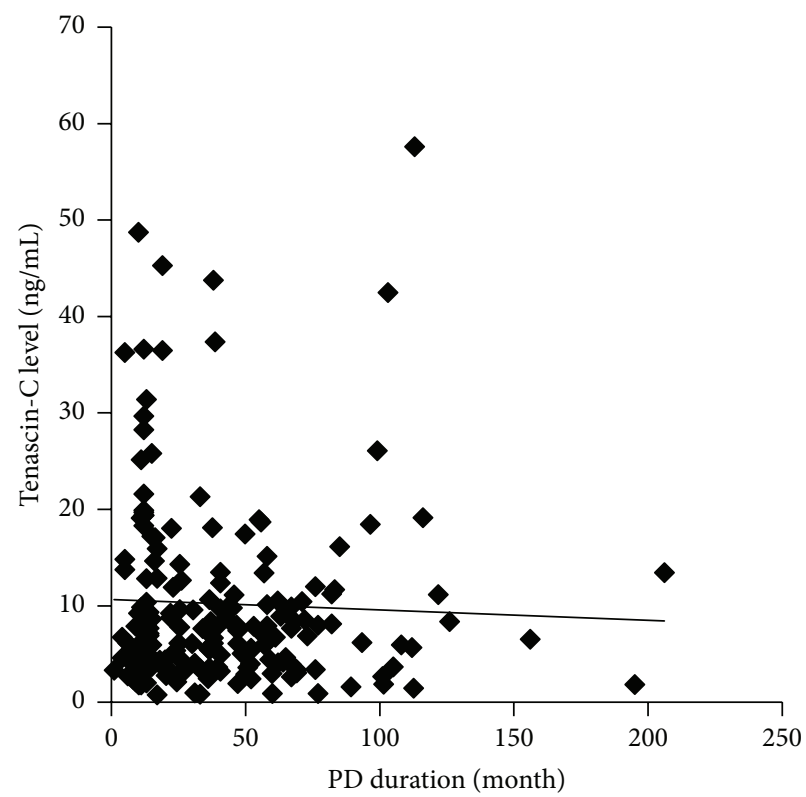

FIGURE 4: The relationship between PD duration and TN-C levels in the drained dialysate.

deterioration using the GDPs-treated rats [12-14]. In the present study, the PD fluids containing $20 \mathrm{mM} \mathrm{MGO}$ or FA were injected to rat. On the other hand, the concentrations of MGO and FA are 2 to $33 \mu \mathrm{M}$ and 6 to $11 \mu \mathrm{M}$, respectively, in conventional commercial PD fluids [22-24]. The MGO and FA concentrations administered to rats were $1,000-10,000$ times higher than those found in commercial PD fluids. We previously confirmed the permitted daily exposure for GDPs concentrations administered to rats in the recommended guideline by the International Conference on Harmonisation (Guideline for Residual Solvents Q3C, 1997) [12, 13]. Moreover, conventional commercial PD fluids contain various toxic GDPs, such as glyoxal, 3-DG, and 3,4-DGE, in addition to MGO and FA. Oh et al. showed in a cell culture experiment with human peritoneal mesothelial cells that $15 \mu \mathrm{M}$ MGO changed the expression of EMT markers, E-cadherin and $\alpha$ smooth muscle actin, and in rat animal models that $27.3 \mu \mathrm{M}$ MGO in combination with other GDPs, such as glyoxal, FA, and 3-DG, induced EMT-like change in mesothelial cells [25]. These findings suggest that the morphological 


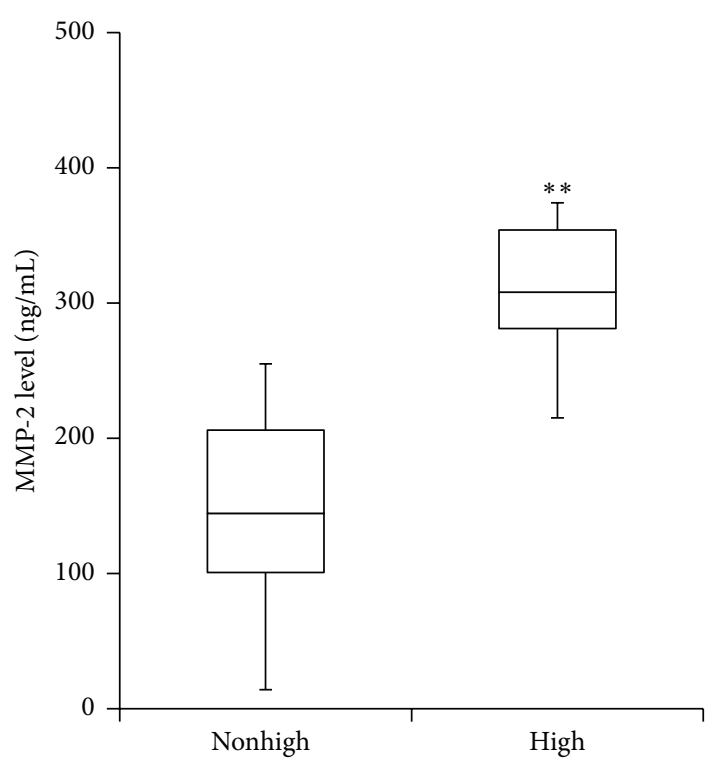

(a)

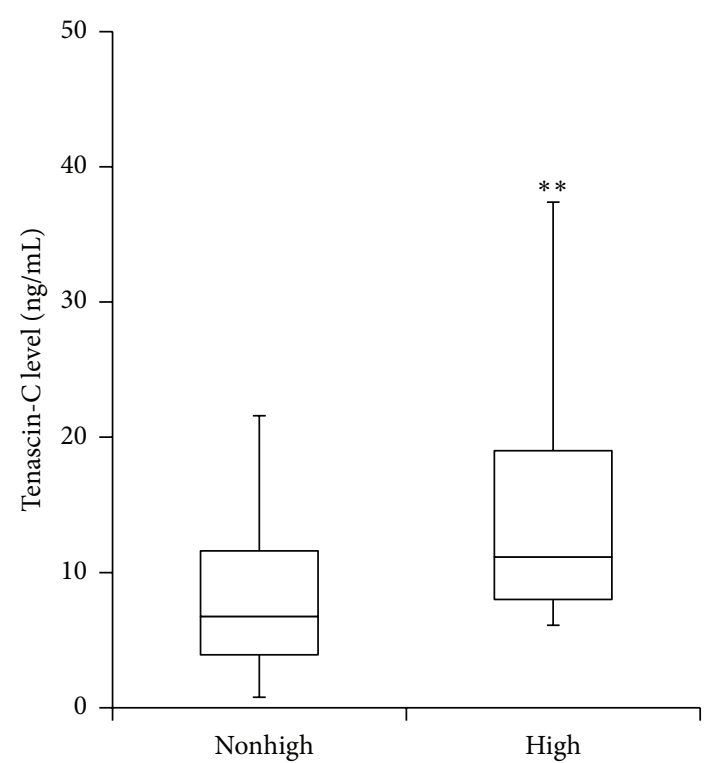

(b)

FIGURE 5: The effluent biomarker levels of patients in the high category of the PET. (a) Effluent TN-C levels. (b) Effluent MMP-2 levels. Data are shown as medians with the spread from 25 th to 75 th percentile (box). ${ }^{* *} p<0.01$ compared with the nonhigh category group.

TABLE 3: The correlation coefficients between patient characteristics and effluent biomarker levels.

\begin{tabular}{lcr}
\hline & \multicolumn{2}{c}{ Biomarker levels in the peritoneal effluents } \\
& Tenascin-C & Matrix metalloproteinase-2 \\
\hline Sex (male/female) & $p=0.62$ & $p=0.50$ \\
Etiology (nondiabetes/diabetes) & $p=0.12$ & $p=0.30$ \\
Age (years) & $\rho=0.048, p=0.51$ & $\rho=0.026, p=0.73$ \\
PD duration (months) & $\rho=-0.070, p=0.34$ & $\rho=0.017, p=0.82$ \\
Peritonitis episode (times) & $\rho=0.15, p<0.05$ & $\rho=0.21, p<0.01$ \\
\hline
\end{tabular}

$\rho$ values: Spearman's correlation coefficient.

TABLE 4: Test performance of effluent biomarker cut-off levels to diagnose the high category of the PET.

\begin{tabular}{|c|c|c|c|c|c|}
\hline Biomarker & Cut-off level & Sensitivity & Specificity & False negative & AUC \\
\hline \multirow{3}{*}{ Tenascin-C } & $5.7 \mathrm{ng} / \mathrm{mL}$ & $100 \%$ & $42 \%$ & $0 \%$ & \multirow{3}{*}{0.71} \\
\hline & $6.0 \mathrm{ng} / \mathrm{mL}$ & $92 \%$ & $43 \%$ & $1.3 \%$ & \\
\hline & $6.5 \mathrm{ng} / \mathrm{mL}$ & $85 \%$ & $48 \%$ & $3.6 \%$ & \\
\hline \multirow{3}{*}{ Matrix metalloproteinase- 2} & $213 \mathrm{ng} / \mathrm{mL}$ & $93 \%$ & $79 \%$ & $0.8 \%$ & \multirow{3}{*}{0.91} \\
\hline & $250 \mathrm{ng} / \mathrm{mL}$ & $86 \%$ & $87 \%$ & $1.4 \%$ & \\
\hline & $270 \mathrm{ng} / \mathrm{mL}$ & $79 \%$ & $88 \%$ & $2.0 \%$ & \\
\hline
\end{tabular}

AUC: area under curve.

changes induced in the present animal experiment can be extrapolated to clinical settings.

In the present and our previous animal studies, MGO and FA resulted in peritoneal deterioration with structural changes and functional decline [12-14]. In the MGO-treated rats, effluent $\mathrm{TN}-\mathrm{C}$ levels were extremely high and, at the surface of the peritoneum where collagen was scarce, TNC-positive mesenchymal-like cells markedly proliferated. We previously reported the possibility that these cells were transformed from mesothelial cells, such as by EMT-like change via TGF- $\beta 1$ [13]. Liu et al. showed that miR-30b is involved in MGO-induced EMT of peritoneal mesothelial cells in rats; miR-30b directly inhibited bone morphogenetic protein-7 (BMP-7) by binding to its $3^{\prime}$-untranslated region, causing unavailability of BMP-7 that might be the antagonist of TGF$\beta 1$-induced EMT [26]. From these reports, MGO may induce EMT-like change in mesothelial cells via TGF- $\beta 1$. Che et al. reported that MGO and 3-DG, reactive dicarbonyl metabolites in the glyoxalase system and glycation reaction, respectively, selectively induced heparin-binding epidermal growth 
factor-like growth factor (HB-EGF) in a dose- and timedependent manner by increasing the intracellular peroxide levels in rat aortic smooth muscle cells (SMC) [27]. However, platelet-derived growth factor, another known growth factor of SMC, was not induced by both dicarbonyls. In addition, the signal transduction by MGO and 3-DG was not mediated by protein kinase C. Stoll et al. demonstrated that expression of HB-EGF increased keratinocyte migration and invasiveness in monolayer culture [28]. Coincident with these changes, HB-EGF significantly decreased several epithelial markers including keratins $1,5,10$, and 14 while increasing expression of markers of cellular motility including SNAIl, ZEB1, COX-2, and MMP-1. HB-EGF induced expression of the mesenchymal protein vimentin and decreased expression of E-cadherin, as well as nuclear translocation of $\beta$-catenin. They also showed that HB-EGF was strongly induced in regenerating the epidermis after partial-thickness wounding of human skin [28]. Taken together, their data suggested that expression of HB-EGF in human keratinocytes triggered a migratory and invasive phenotype with many features of EMT. From these reports, MGO may induce EMT-like change in mesothelial cells via HB-EGF.

MGO is a potent promoter of the production of advanced glycation end-products (AGEs). In a previous study using rats, the administration of $20 \mathrm{mM}$ MGO resulted in the accumulation of AGEs, such as imidazolone and carboxyethyl lysine, in the peritoneum [29]. AGEs and MGO induced the expression of the receptor for AGE (RAGE), and then AGERAGE interaction induced EMT-like change in mesothelial cells via upregulation of expression of transforming growth factor- $\beta$ (TGF- $\beta$ ) and vascular endothelial growth factor (VEGF) $[24,30,31]$. We have previously showed that, in $20 \mathrm{mM}$ MGO-treated rats, AGE was detected in mesothelial cells with EMT-like change at the surface of the peritoneum and the gene expressions of RAGE, TGF- $\beta$, and VEGF were enhanced in the peritoneum [12, 13]. Thus, EMT-like change in mesothelial cells was induced in response to signals from RAGE binding with AGE. These reports suggest that MGO induces EMT-like change in mesothelial cells not only directly but also indirectly via formation of AGEs.

In the FA-treated rats, peritoneal injury with cellular infiltration was induced; however, cells were lost at the surface of the peritoneum. The peritoneum was fibrous thickening with dense collagen fibers [12-14]. The pathological picture of the peritoneum of the FA-treated rats resembles that of the chlorhexidine gluconate-treated rats $[13,14]$. These peritoneal changes may be generally induced by toxic agents.

Effluent TN-C levels in the FA-treated rats increased but were only approximately one-tenth of the levels of the MGOtreated rats. In contrast, effluent levels of MMP-2, a biomarker of peritoneal injury, were extremely high in both MGO- and FA-treated rats. From these results, the effluent TN-C levels reflected not only peritoneal deterioration but also especially EMT-like change in mesothelial cells in the animal study.

In the present clinical study, the measured $\mathrm{D} / \mathrm{S}$ ratio of $\mathrm{TN}-\mathrm{C}$ was significantly higher than the expected ratio if TN$\mathrm{C}$ in the effluent was transported only from the circulation. The difference between the measured $\mathrm{D} / \mathrm{S}$ ratio and the expected ratio may be attributable to the local production of TN-C in the peritoneal tissue rather than the transport of TN-C from the circulation [8-10]. In addition, in the present animal study, mesothelial cells with EMT-like change that proliferated at the surface of the peritoneum showed positive signals on immunohistochemical analysis using the anti-TN$\mathrm{C}$ antibody. Most TN-C in the drained dialysate may be produced from these cells in the peritoneum and effluent TN-C levels may reflect EMT-like change in mesothelial cells. From these results, effluent $\mathrm{TN}-\mathrm{C}$ could be an effective diagnostic indicator of peritoneal deterioration with structural changes, particularly of mesothelial cells with EMT-like change. To confirm this hypothesis, in a clinical study we should examine the correlation between the effluent TN-C levels and EMTlike change in mesothelial cells by cytopathological analysis using biopsy of peritoneum. However, this would have been difficult to conduct in the present study because of invasive sampling.

In the present multicenter clinical study, the peritoneal transport rate estimated by the PET closely correlated with effluent TN-C levels; however, the correlation coefficient of the TN-C levels was lower than that with the MMP-2 levels. Effluent MMP-2 levels were able to predict, with high sensitivity and specificity, the high category of the PET, which is a risk factor for EPS [5]. In our previous clinical studies, effluent MMP-2 levels were high in patients with peritoneal injury [7]. In many animal studies, effluent MMP-2 levels increased in various peritoneal injury animal models with or without EMT-like change in mesothelial cells [9, 12, 13, 25]. We suggest that MMP-2 should serve as a superior indicator of general peritoneal deterioration.

In the present clinical study, the correlation coefficient between the effluent levels of TN-C and MMP-2 was high. MMP-2 and the degradation of TN-C are associated with tumor recurrence in early-stage non-small cell lung cancer [32]. TN-C deposition into the extracellular matrix requires the participation of MMP-2 and the resulting deposited TN$\mathrm{C}$ promotes pancreatic cancer progression [33]. In peritoneal deterioration, $\mathrm{TN}-\mathrm{C}$ may promote tissue injury in concert with MMP-2.

Effluent TN-C levels correlated with the peritoneal solute transport rate, but not PD duration in the present crosssectional study. Confirmation that the change in effluent TN-C levels reflects increased peritoneal solute transport or EMT-like change in mesothelial cells is necessary to reach the conclusion that TN-C is a useful biomarker for earlystage peritoneal deterioration. In our longitudinal analysis, peritoneal solute transport did not develop significantly faster during the period of the present study. Further longitudinal analysis is needed.

\section{Conclusion}

Effluent TN-C levels correlated with the peritoneal solute transport rate. Most TN-C in the drained dialysate is thought to be produced by mesothelial cell-derived mesenchymal cells at the surface of the peritoneum. Therefore, effluent TN-C has a possibility of biomarker for EMT-like change in mesothelial cells in PD. Because EMT-like change in mesothelial cells is thought to be a trigger of peritoneal injury, TN-C may be 
useful as an indicator for early-stage peritoneal deterioration, whereas effluent MMP-2 levels reflect general peritoneal deterioration. To test this hypothesis, further longitudinal studies are necessary to examine the change in effluent TN-C levels during the progression of peritoneal deterioration with increased peritoneal solute transport.

\section{Disclosure}

Part of this study was presented at the 18th annual meeting of the Japanese Society for Peritoneal Dialysis held at Osaka, Japan (September 2013).

\section{Conflict of Interests}

Ichirou Hirahara is affiliated with Terumo Core Technology Center (Kanagawa, Japan).

\section{Acknowledgments}

The collaborators in the multicenter clinical study were as follows: Fumihiko Hatafuku (Department of Urology, Onoda Hospital), Hideki Takizawa (Department of Nephrology, Teine Keijinkai Hospital), Makoto Nishina (Metabolism, Department of Internal Medicine, Tokai University, School of Medicine), Masanobu Horie (Department of Urology, Daiyukaidaiichi Hospital), Morihiro Kondou (Department of Nephrology, Otowa Hospital), Naomi Yoshimune (Department of Internal Medicine, Kinashi Obayashi Hospital), Noriaki Yorioka (Department of Advanced Nephrology, Graduate School of Biomedical Sciences, Hiroshima University), Ryoichi Miyazaki (Department of Internal Medicine, Fujita Memorial Hospital), Ryouji Wakamatsu (Nishikatakai Clinic), Sukenari Koyabu (Department of Internal Medicine, Owase General Hospital), Tadashi Yamamoto (Kidney Center, Shirasagi Hospital), Takeyuki Hiramatsu (Department of Internal Medicine, Aihoku Hospital), Tetsurou Yanase (Dr. Yanase Internal Medicine Office), Tohru Mizumasa (Department of Nephrology, Fukuoka Red Cross Hospital), Tomoyoshi Kimura (Department of Nephrology, Sendai Social Insurance Hospital), Toyonori Saiki (Department of Nephrology, Saiki Jin Clinic), and Yumiko Ikeda (Department of Nephrology, Yokohama Minami Kyousai Hospital). The authors would like to thank them for their help with the preparation of clinical samples and valuable discussions. This study was supported by Terumo Core Technology Center (Kanagawa, Japan). The authors would like to thank Ms. Yuka Shouji (Terumo Core Technology Center) for her assistance with the preparation of pathological tissue, Dr. Taizo Iwasaki (Terumo Core Technology Center) for his help with statistical analysis, and Professor Toshimichi Yoshida (Departments of Pathology and Matrix Biology, Mie University, Mie, Japan) for valuable discussions.

\section{References}

[1] V. C. Gandhi, H. M. Humayun, T. S. Ing et al., "Sclerotic thickening of the peritoneal membrane in maintenance peritoneal dialysis patients," Archives of Internal Medicine, vol. 140, no. 9, pp. 1201-1203, 1980.

[2] D. W. Schmidt and M. F. Flessner, "Pathogenesis and treatment of encapsulating peritoneal sclerosis: basic and translational research," Peritoneal Dialysis International, vol. 28, supplement 5, pp. S10-S15, 2008.

[3] Y. Kawaguchi, A. Saito, H. Kawanishi et al., "Recommendations on the management of encapsulating peritoneal sclerosis in Japan, 2005: diagnosis, predictive markers, treatment, and preventive measures," Peritoneal Dialysis International, vol. 25, supplement 4, pp. S83-S95, 2005.

[4] Working Group Committee for the Preparation of Guidelines for Peritoneal Dialysis, "Japanese Society for Dialysis Therapy. 2009 Japanese society for dialysis therapy guidelines for peritoneal dialysis," Therapeutic Apheresis Dialysis, vol. 14, no. 6, pp. 489-504, 2010.

[5] R. Yamamoto, Y. Otsuka, M. Nakayama et al., "Risk factors for encapsulating peritoneal sclerosis in patients who have experienced peritoneal dialysis treatment," Clinical \& Experimental Nephrology, vol. 9, no. 2, pp. 148-152, 2005.

[6] Z. J. Twardowski, K. D. Nolph, R. Khanna et al., "Peritoneal equilibration test," Peritoneal Dialysis Bulletin, vol. 7, no. 3, pp. 138-147, 1987.

[7] I. Hirahara, M. Inoue, K. Okuda, Y. Ando, S. Muto, and E. Kusano, "The potential of matrix metalloproteinase- 2 as a marker of peritoneal injury, increased solute transport, or progression to encapsulating peritoneal sclerosis during peritoneal dialysis-a multicentre study in Japan," Nephrology Dialysis Transplantation, vol. 22, no. 2, pp. 560-567, 2007.

[8] I. Hirahara, M. Inoue, T. Umino, O. Saito, S. Muto, and E. Kusano, "Matrix metalloproteinase levels in the drained dialysate reflect the peritoneal solute transport rate: a multicentre study in Japan," Nephrology Dialysis Transplantation, vol. 26, no. 5, pp. 1695-1701, 2011.

[9] D. L. Barreto, A. M. Coester, D. G. Struijk, and R. T. Krediet, "Can effluent matrix metalloproteinase 2 and plasminogen activator inhibitor 1 be used as biomarkers of peritoneal membrane alterations in peritoneal dialysis patients?" Peritoneal Dialysis International, vol. 33, no. 5, pp. 529-537, 2013.

[10] M. M. Zweers, D. R. de Waart, W. Smit, D. G. Struijk, and R. T. Krediet, "Growth factors VEGF and TGF- $\beta 1$ in peritoneal dialysis," The Journal of Laboratory and Clinical Medicine, vol. 134, no. 2, pp. 124-132, 1999.

[11] Y. Kaku, K. Nohara, Y. Tsutsumi et al., “The relationship among the markers of peritoneal function such as PET, MMP-2, IL-6 etc, in pediatric and adolescent PD patients," Jin To Touseki, vol. 57, supplement, pp. 296-298, 2004.

[12] I. Hirahara, E. Kusano, S. Yanagiba et al., "Peritoneal injury by methylglyoxal in peritoneal dialysis," Peritoneal Dialysis International, vol. 26, no. 3, pp. 380-392, 2006.

[13] I. Hirahara, Y. Ishibashi, S. Kaname, E. Kusano, and T. Fujita, "Methylglyoxal induces peritoneal thickening by mesenchymallike mesothelial cells in rats," Nephrology Dialysis Transplantation, vol. 24, no. 2, pp. 437-447, 2009.

[14] I. Hirahara, H. Sato, T. Imai et al., "Methylglyoxal induced basophilic spindle cells with podoplanin at the surface of peritoneum in rat peritoneal dialysis model," BioMed Research International, vol. 2015, Article ID 289751, 7 pages, 2015.

[15] S. Osada, C. Hamada, T. Shimaoka, K. Kaneko, S. Horikoshi, and Y. Tomino, "Alterations in proteoglycan components and histopathology of the peritoneum in uraemic and peritoneal 
dialysis (PD) patients," Nephrology Dialysis Transplantation, vol. 24, no. 11, pp. 3504-3512, 2009.

[16] M. Yáñez-Mó, E. Lara-Pezzi, R. Selgas et al., "Peritoneal dialysis and epithelial-to-mesenchymal transition of mesothelial cells," The New England Journal of Medicine, vol. 348, no. 5, pp. 403413, 2003.

[17] A. Aguilera, M. Yáñez-Mo, R. Selgas, F. Sánchez-Madrid, and M. López-Cabrera, "Epithelial to mesenchymal transition as a triggering factor of peritoneal membrane fibrosis and angiogenesis in peritoneal dialysis patients," Current Opinion in Investigational Drugs, vol. 6, no. 3, pp. 262-268, 2005.

[18] G. del Peso, J. A. Jiménez-Heffernan, M. A. Bajo et al., "Epithelial-to-mesenchymal transition of mesothelial cells is an early event during peritoneal dialysis and is associated with high peritoneal transport," Kidney International, vol. 73, supplement, pp. S26-S33, 2008.

[19] K. Imanaka-Yoshida, M. Hiroe, Y. Yasutomi et al., "Tenascin$\mathrm{C}$ is a useful marker for disease activity in myocarditis," The Journal of Pathology, vol. 197, no. 3, pp. 388-394, 2002.

[20] K. Nagaharu, X. Zhang, T. Yoshida et al., “Tenascin C induces epithelial-mesenchymal transition-like change accompanied by SRC activation and focal adhesion kinase phosphorylation in human breast cancer cells," American Journal of Pathology, vol. 178, no. 2, pp. 754-763, 2011.

[21] D. Katoh, K. Nagaharu, N. Shimojo et al., "Binding of $\alpha v \beta 1$ and $\alpha \mathrm{v} \beta 6$ integrins to tenascin- $C$ induces EMT like change of breast cancer cell," Oncogenesis, vol. 19, no. 2, p. e65, 2013.

[22] C. B. Nilsson-Thorell, N. Muscalu, A. H. G. Andren, P. T. T. Kjellstrand, and A. P. Wieslander, "Heat sterilization of fluids for peritoneal dialysis gives rise to aldehydes," Peritoneal Dialysis International, vol. 13, no. 3, pp. 208-213, 1993.

[23] A. P. Wieslander, R. Deppisch, E. Svensson, G. Forsback, R. Speidel, and B. Rippe, "In vitro biocompatibility of a heatsterilized, low-toxic, and less acidic fluid for peritoneal dialysis," Peritoneal Dialysis International, vol. 15, no. 3, pp. 158-164, 1995.

[24] K. N. Lai, J. C. K. Leung, L. Y. Y. Chan et al., "Differential expression of receptors for advanced glycation end-products in peritoneal mesothelial cells exposed to glucose degradation products," Clinical \& Experimental Immunology, vol. 138, no. 3, pp. 466-475, 2004.

[25] E.-J. Oh, H.-M. Ryu, S.-Y. Choi et al., "Impact of low glucose degradation product bicarbonate/lactate-buffered dialysis solution on the epithelial-mesenchymal transition of peritoneum," American Journal of Nephrology, vol. 31, no. 1, pp. 58-67, 2009.

[26] H. Liu, N. Zhang, and D. Tian, "MiR-30b is involved in methylglyoxal-induced epithelial-mesenchymal transition of peritoneal mesothelial cells in rats," Cellular \& Molecular Biology Letters, vol. 19, no. 2, pp. 315-329, 2014.

[27] W. Che, M. Asahi, M. Takahashi et al., "Selective induction of heparin-binding epidermal growth factor-like growth factor by methylglyoxal and 3-deoxyglucosone in rat aortic smooth muscle cells: the involvement of reactive oxygen species formation and a possible implication for atherogenesis in diabetes," The Journal of Biological Chemistry, vol. 272, no. 29, pp. 18453-18459, 1997.

[28] S. W. Stoll, L. Rittié, J. L. Johnson, and J. T. Elder, "Heparinbinding EGF-like growth factor promotes epithelialmesenchymal transition in human keratinocytes," Journal of Investigative Dermatology, vol. 132, no. 9, pp. 2148-2157, 2012.

[29] M. Nakayama, A. Sakai, M. Numata, and T. Hosoya, "Hypervascular change and formation of advanced glycation endproducts in the peritoneum caused by methylglyoxal and the effect of an anti-oxidant, sodium sulfite," American Journal of Nephrology, vol. 23, no. 6, pp. 390-394, 2003.

[30] V. Schwenger, C. Morath, A. Salava et al., "Damage to the peritoneal membrane by glucose degradation products is mediated by the receptor for advanced glycation end-products," Journal of the American Society of Nephrology, vol. 17, no. 1, pp. 199-207, 2006.

[31] A. S. De Vriese, R. G. Tilton, S. Mortier, and N. H. Lameire, "Myofibroblast transdifferentiation of mesothelial cells is mediated by RAGE and contributes to peritoneal fibrosis in uraemia," Nephrology Dialysis Transplantation, vol. 21, no. 9, pp. 25492555,2006

[32] M. Cai, K. Onoda, M. Takao et al., "Degradation of tenascin-C and activity of matrix metalloproteinase- 2 are associated with tumor recurrence in early stage non-small cell lung cancer," Clinical Cancer Research, vol. 8, no. 4, pp. 1152-1156, 2002.

[33] J. Chen, Z. Chen, M. Chen et al., "Role of fibrillar tenascin-C in metastatic pancreatic cancer," International Journal of Oncology, vol. 34, no. 4, pp. 1029-1036, 2009. 


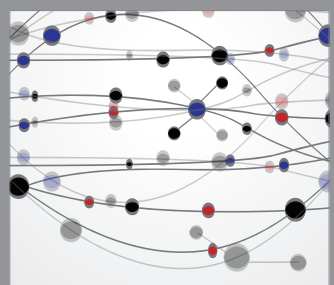

The Scientific World Journal
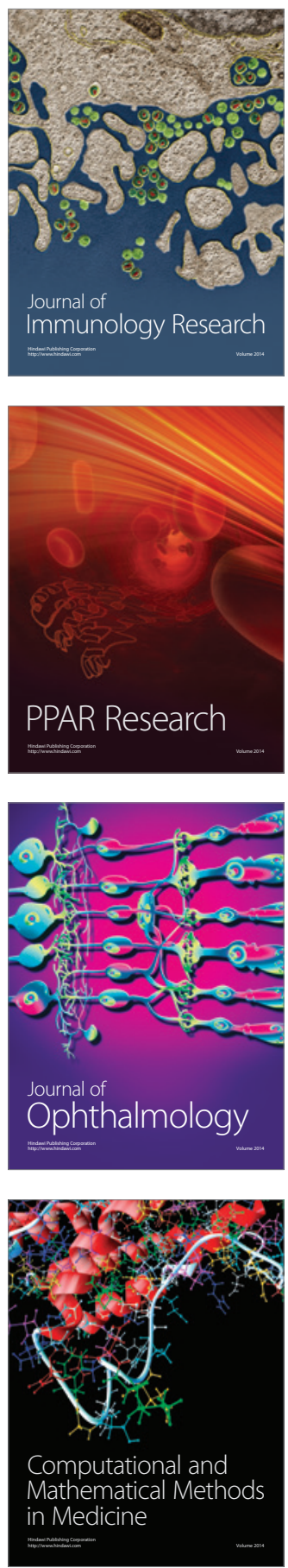

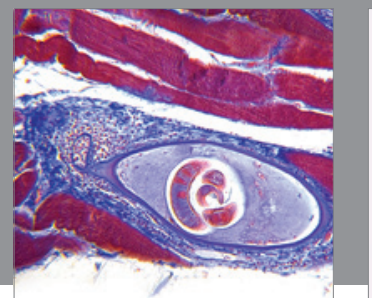

Gastroenterology

Research and Practice
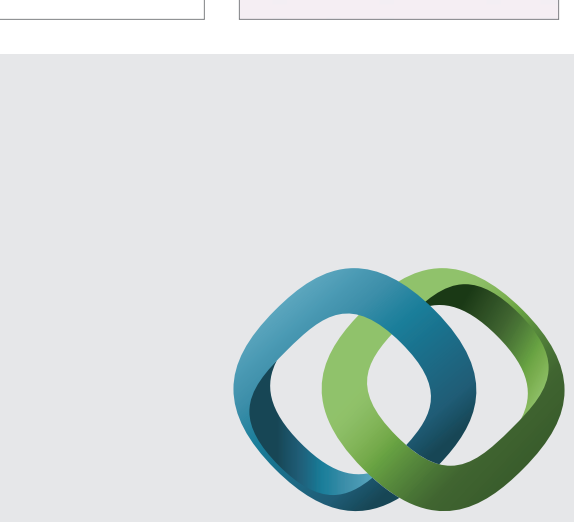

\section{Hindawi}

Submit your manuscripts at

http://www.hindawi.com
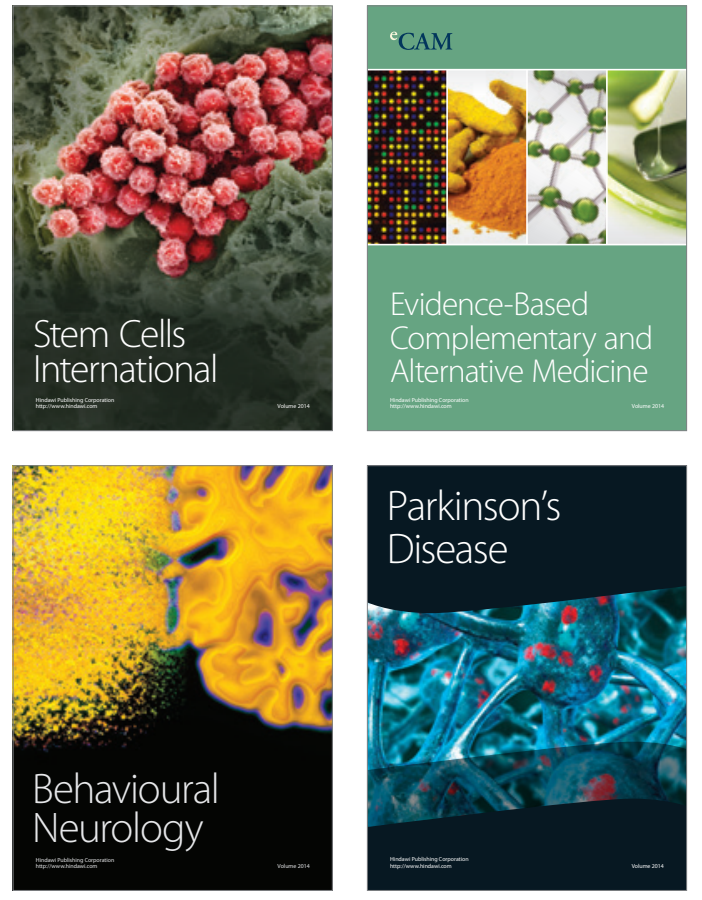
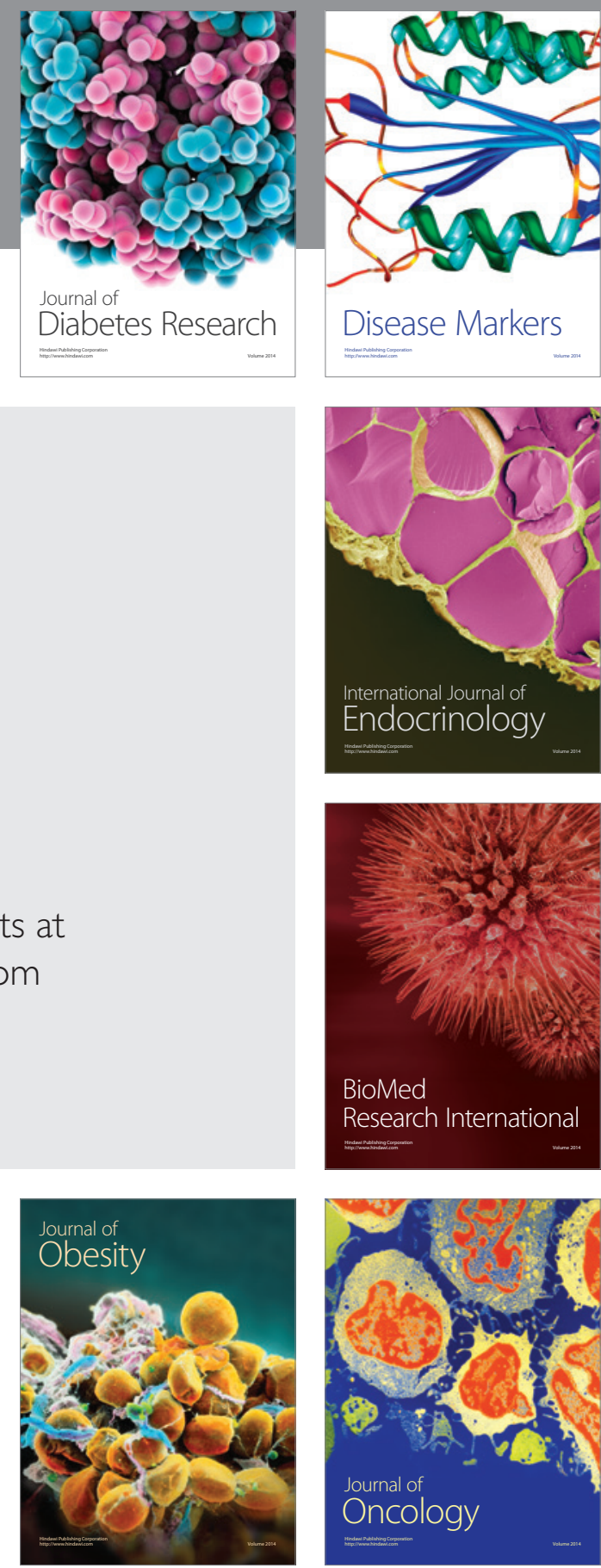

Disease Markers
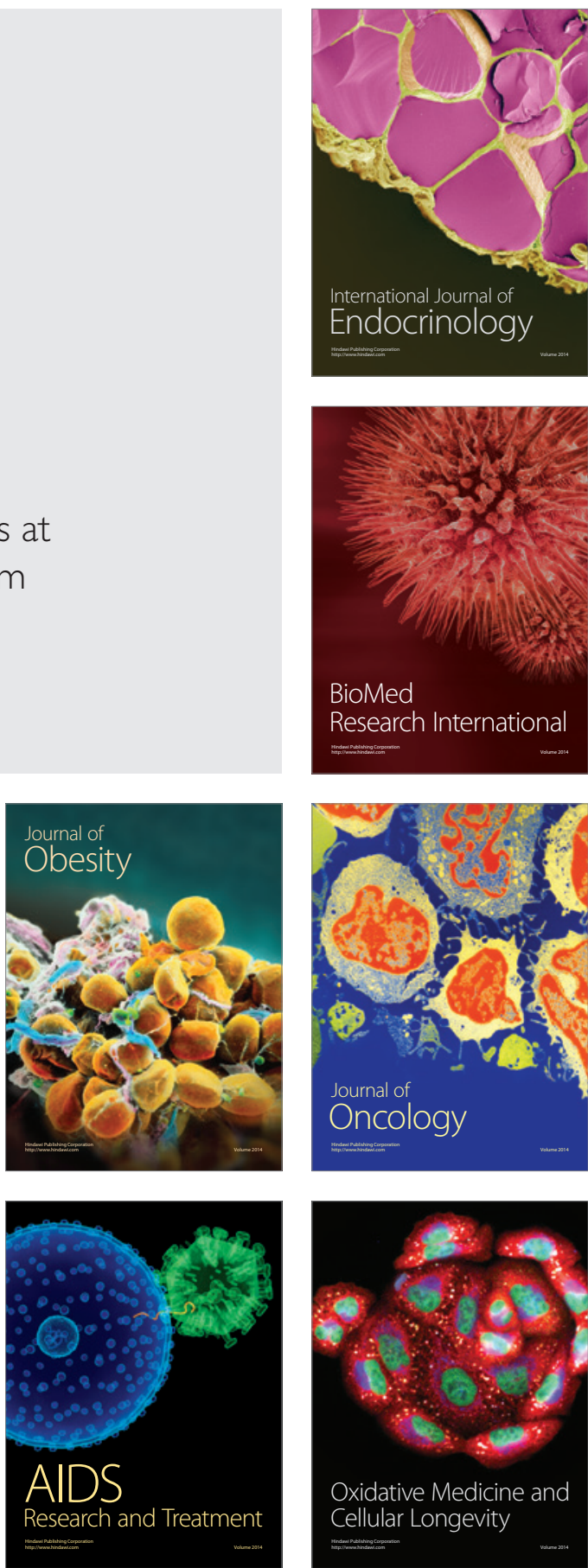